\author{
DAVID NACCACHE \\ PHILIPS TRT, SMART-CARDS \& SYSTEMS, BPI 95, \\ 5 AVE. REAUMUR, BP 21, F 92352, LE PLESSIS ROBINSON CEDEX, FRANCE. \\ EMAIL : NACCACH1@FRPRSOCS.SNADS.PHILIPS.NL
}

\begin{abstract}
Montgomery's algorithm [2] is a process for computing A B $2^{-|n|}$ modulo $n$ in $O(\log (n))$ memory space.

Here we construct a Fiat-Shamir-like authentication scheme [1] suitable for Montgomery environnments without introducing any overhead in the number of modular multiplications requested for the execution of the normal protocol.
\end{abstract}

A very recent result [3] establishes (in a constructive way) that A B $2^{-|n|}$ arod $n$ can be computed with the same complexity (timewise and hardwarewise) as $A$ (not mod $n$ ).

This theoretical reduction of the problem of modular multiplication, recently applied to the design of today's fastest hardware modular multiplier, is very important since it implies that the protocol presented hereafter can be executed in the same time as a Fiat-shamir where all modular multiplications are replaced by standard multiplications.

The fact that no constants are to be precalculated beforehand and the small amount of RAM requested for software implementation of the new protocol makes it highly convenient for smart-card applications.

\title{
INTRODUCTION
}

All along this paper, $|n|$ denotes the length of $n$ (in bits) and |n| the Hamming weight of $n$.

Montgomery's algorithm for modular multiplication [2] is a process for computing $A$ B $2^{-|n|}$ modulo $n$ in $O(\log (n))$ memory space.

The method only assumes that $n$ is odd. No other restrictions are imposed on the modulus. 
Very much simplified, this algorithm works as follows: Let $x[i]$ denote $x^{\prime} s i^{\text {th }}$ bit (with $x[0]$ as LSB) and $K={ }_{4}|n| \bmod n$. Kernel Algorithm(A, B)

\{

$c=0$

For $i=0$ to $|n|-1$

If $A[i]=1$ then $C=C+B$

If $c[i]=1$ then $c=c+n$

$c=c / 2$

If $c=n$ then $c=c-n$

return(c)

r

It can be shown [1] that $c=A$ B $2^{-|n|} \bmod n$ and that : $\operatorname{Kernel}(K, \operatorname{Kernel}(A, B))=A B \bmod n$

$A$ more comprehensive and complete approach is presented by Arazi in [3].

THE NEW FIAT-SHAMIR-LIKE PROTOCOL.

(3) formally shows how to reduce the complexity of the computation Kernel (A, B) into that of $A$ B.

From this remark and the last section it clearly appears that if it is possible to transform the Fiat-Shamir scheme in such a way that the parasites $\left(2^{-|n|}\right.$ ) will not disturb the protocol (using only one Kernel operation for each modular multiplication), then it would be possible to perform the Fiat-shamir in about the half of the time requested with a full Montgomery multiplier.

Moreover, we do not require the precalculation or usage of the constant $K$.

This new approach to the problem is somewhat new since by opposition to the classical process of designing computationnal tools for comfortable execution of number-theoretic protocols, here we transform a cryptographic scheme in order to meet a given computationnal limitation. 
Actualiy, after a proper modiflcation of the relation between the public and the secret keys, we will see that not only the Montgonery parasites don't disturb, they even help I (As ald by Abraham Lincoln: "The best way to destroy your enemies is to transform thea into friends").

From now on we will denote by 0 the parasite factor $2^{-|n|}$ mod $n$ and assume the availibility of a half Montgomery multipliar (that is a Kernel procedure performing only the operation $A D \bmod n$ ).

\section{THE NEW PROTOCOL}

Redef ine the Fiat-Shamir (1) public $v_{j}-s$ by $: D^{3} v_{j} s_{j}^{2}$ - 1 mod $n$ And assume that the $v_{j}$ 's are already known to the verifier (for instance by $F$ iat and Shamir's $F(I D, j)$ method).

\section{sten $\lambda$}

The prover computes $z=R^{2} D$ mod $n-\operatorname{kernel}(R, R)$ and sends it to the variflar.

\section{Step 2}

The varlfler sends the random binary vector $a$.

\section{Stan 2}

The prover computes and sends $y=r \prod_{i-1} s_{i}|e| \bmod n$.

This value is easily computed by :

$y=\operatorname{Karnal}\left(s_{i_{1}}, \operatorname{Karnel}\left(s_{i_{2}}, \ldots \operatorname{Karnal}\left(s_{1}{ }_{|e|}, r\right)\right) \ldots\right)$

Here the $i_{j}$-s anote the $|e|$ indices selected by vector $e$.

\section{Step 4}

The verifier computes (similar way to that of the previous step) :

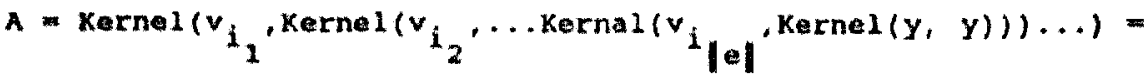
$y^{2} D \prod_{i} v_{1} d^{|e|-1} 0 \bmod n-x^{2} \prod_{i=1} v_{i} s_{i}^{2} D^{2|a|}$ dal $D \bmod n=$ 
$r^{2} \prod_{i=1} v_{j} s_{j}^{2} D^{3|e|} D \bmod n=r^{2} D \bmod n$. And tests if $A \stackrel{?}{=} z$.

similarly, this can be applied to the digital signature as well.

CONCLUSION AND IMPLEMENTATION DETAILS.

With a 68HC05 running at $3.5 \mathrm{MHz}$ the Kernel operation for 512 bit numbers) was implemented in less than $135 \mathrm{~ms}$, RAM usage is less than 70 bytes. A special Kernel-Squaring version runs at 85 ms but requires a double RAM space.

In [3] it is shown that "It is possible to compute $A B D$ mod $n$ in $|n|+1$ clock cycles. That is, a modular multiplication is performed with the same complexity (timewise and hardwarewise) as that of a standard multiplication operation", it is thus possible to execute a Fiat-Shamir identity check (and signature, with similar modifications of the scheme) in hardware and time equivalent to that required for the execution of the protocol without modular reductions.

The same strategy of modifying the relationship between public and secret keys in order to meet or cancel the effect of parasite constants introduced by modular reduction tools can be applied to a big variety of number-theoretic authentication and signature protocols.

\section{REFERENCES}

[1] Fiat Feige and Shamir, "Zero-Knowledge Proofs of Identity", J. Cryptology, vol 1, pp. 77-94.

[2] Montgomery, "Modular Multiplication without Trial Division", Mathematics of Computation, vol 44, pp 519-521.

[3] Arazi, "Modular Multiplication is Equivalent in Complexity to a standard Multiplication", Fortress U\&T Internal Report (1992) available from Fortress U\&T Information Safeguards, P.0. Box 1350, Beer-Sheva, IL-84110, Israel. 